\title{
Computational Study of Compact Ejector-Enhanced Resonant Pulse Combustors
}

\author{
Shaye Yungster* \\ Ohio Aerospace Institute, Cleveland, OH 44142 \\ Daniel E. Paxson ${ }^{\dagger}$ and Hugh D. Perkins $\$$ \\ NASA Glenn Research Center, Cleveland, OH 44135
}

\begin{abstract}
Previous studies of Ejector-Enhanced Resonant Pulse Combustors considered configurations that were relatively long, making them difficult to incorporate in practical gas turbine engines. In the present study, more compact configurations are analyzed, focusing on the system pressure gain. The study shows that it is possible to reduce the length of both the pulse combustor and ejector components without compromising the device's performance. In fact, it is found that in several of the compact configurations analyzed, the system pressure gain actually increased, reaching pressure gain levels above $5 \%$, significantly higher than those obtained in previous studies. The Rayleigh efficiency, which has been used in the past to characterize the performance of pulse combustors, is computed for several of the Ejector-Enhanced Resonant Pulse Combustor configurations. The Rayleigh efficiency is seen to correlate with both average combustor pressure and system pressure gain for a given configuration, however, it could not be used to compare different configurations.
\end{abstract}

\section{Nomenclature}

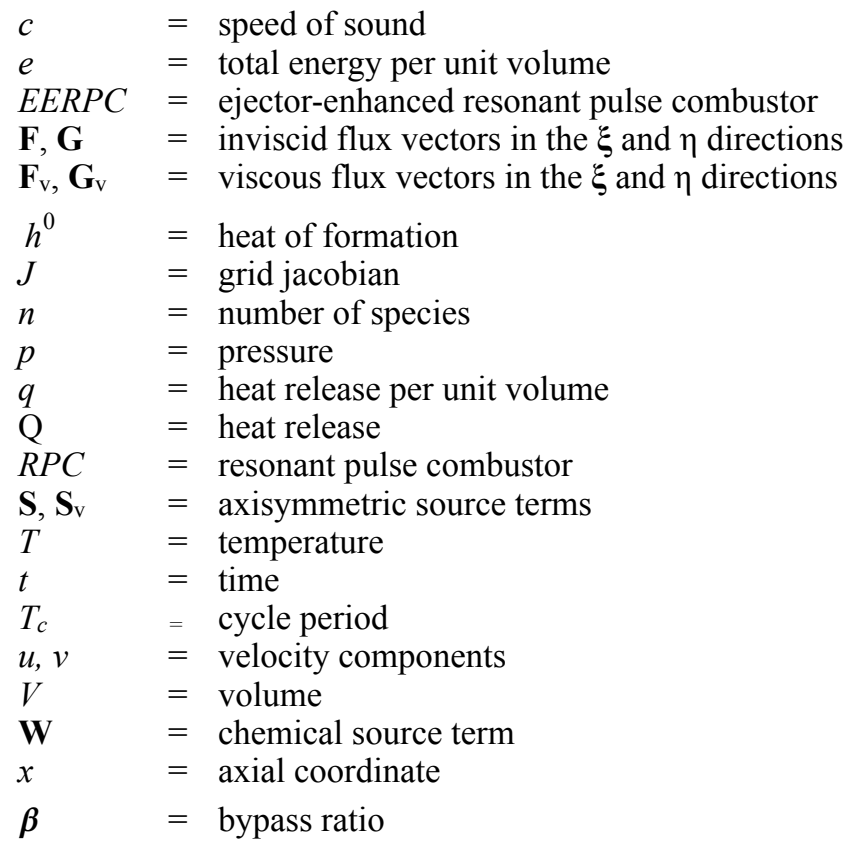

\footnotetext{
* Senior Scientist, Senior AIAA Member.

$\uparrow$ Aerospace Engineer, Associate Fellow AIAA.

$¥$ Aerospace Engineer, AIAA Member.
} 


$\begin{array}{ll}\gamma & =\text { ratio of specific heats } \\ \rho & =\text { density } \\ \phi & =\text { equivalence ratio } \\ \xi, \eta & =\text { generalized coordinates } \\ \eta_{R} & =\text { Rayleigh efficiency }\end{array}$

\begin{tabular}{|c|c|c|}
\hline Super & pts/ & Subscripts \\
\hline$(-)$ & & time-average \\
\hline ( ) avg & $=$ & volumetric average \\
\hline
\end{tabular}

\section{Introduction}

Resonant pulse combustors (RPC) are unsteady thermo-acoustic devices capable of producing pressure gain during combustion by a process of flow confinement, achieved by the synchronized action of the heat release, mechanical valves, and gasdynamic waves, that approximates constant volume combustion. The basic operating principles of RPCs were first explained by Lord Rayleigh ${ }^{1}$ in his 1878 work on combustion instabilities. The notion that a controlled instability could produce useful work was subsequently developed and exploited in various devices. One of the better known early applications of pulse combustion was the V-1 "buzz bomb" used in World War II.

In recent years, interest in pulse combustion for gas turbine engine applications has increased due to its potential for achieving pressure gain during the combustion process. The potential benefits of pressure-gain combustion, in general, have motivated a large number of experimental and analytical investigations of various concepts that include detonation-based devices, wave rotors and RPCs, all of which are unsteady devices.

When used in combination with ejector systems, RPCs have several advantages over the alternative pressure gain combustion concepts mentioned above. They are relatively simple devices, and thereby avoid the mechanical complexities of the other devices. Flow non-uniformities at the exit of Ejector-Enhanced Resonant Pulse Combustors (EERPC) have been shown to be substantially lower in comparison to those observed in detonationbased devices ${ }^{2}$. This smoothing aspect of pulse-combustor-based systems is critical for maintaining high turbomachinery performance. In addition, the emissions of oxides of nitrogen in RPCs are potentially lower than in conventional combustor systems ${ }^{3}$. The main disadvantage of RPC-based systems is that the pressure-gain attainable is typically lower than that for wave rotors or detonation based devices. On the other hand, the lower pressure-gain carries with it the implication of lower thermal and mechanical stresses.

Practical aerospace applications of pulse-combustion-based systems necessitate operation at high-pressure conditions. Initial calculations of EERPC configurations operating at high-pressure conditions (10 bar) produced pressure gains significantly lower than those observed experimentally and computationally at atmospheric conditions ${ }^{4}$. A subsequent study ${ }^{5}$ identified the factors limiting the pressure-gain at high-pressure conditions and investigated the effects of fuel injection and air mixing characteristics on performance. New pulse combustor configurations were developed in Ref. 5 which were able to achieve performance levels at high-pressure conditions comparable to those observed at atmospheric conditions.

The study presented in Ref. 5 only considered the pulse combustor by itself, which is not suitable to replace a conventional combustor in a gas turbine engine since, among other factors, the minimum fuel-to-air ratio for successful RPC operation produces a flow that is too hot for downstream turbomachinery. As such, some amount of bypass air is required.

In recent studies, ${ }^{6,7}$ the performance of various EERPC configurations operating at high-pressure conditions were investigated, focusing on the effects of equivalence ratio and ejector throat area. The philosophy behind this approach was to take advantage of the relatively efficient mixing and pumping characteristics of unsteady ejectors for introducing the bypass flow. The results demonstrated pressure gains of approximately $3 \%$ at gas turbine compatible combustor temperature ratios, with nearly smooth exit flow. Such results were comparable to experiments conducted by Paxson and Dougherty ${ }^{2}$ that demonstrated pressure gains of $3.5 \%$ at sea level static conditions.

Figure 1 shows a schematic of a RPC. The reed valve is the only moving part in the RPC. Its position is determined at every instant by the pressure differential existing between the inlet and combustor sides. Modeling the reed valve action is one of the main challenges of conducting numerical simulations of these devices. 
In the previous studies ${ }^{4-7}$, a vertically sliding valve model (shown in Fig. 2), that approximated the reed valve action, was utilized\$. This valve model worked well and provided insight into the effects of valve aerodynamics. This valve model is also utilized in the present study. Opening and closing of the valve is determined by the pressure at the headend of the combustor. Specifically, valve opening is triggered when the pressure drops below the inlet total pressure, $p_{0}$, and valve closing is triggered when the pressure rises above $p_{0}$ later in the cycle.

Fuel is injected through discrete injectors located inside the inlet just upstream of the valve. The amount of fuel injected in one pulse-combustor cycle can be adjusted by either changing the fuel injection pressure or by changing the fuel injection duration time. This type of pulsed, discrete fuel injection delivery method was utilized by Offord et. al. ${ }^{9}$ in their experimental investigation of a valveless pulse combustor.

In addition, in these previous studies, the pressure gain potential of the EERPC was investigated by applying a specified back pressure at the EERPC exit boundary. While this approach was effective in the numerical simulations, a more realistic model of the EERPC was adopted in Ref. 8. In that model, a converging nozzle is added at the exit of the EERP, to match more closely the effect of inlet guide vanes that are immediately downstream of a gas turbine combustor. The throat area of the exit nozzle can be adjusted to obtain the desired back-pressure level. This model is also utilized in the present study.

One disadvantage of the configurations considered in the previous studies ${ }^{4-8}$ was that the combustors were relatively long, making them difficult to incorporate in a practical gas turbine engine. The length of the pulse combustor was selected based on previous experimental studies ${ }^{2}$ and the length of the ejector was selected to provide complete mixing. In the present study, it is shown that it's possible to reduce the length of both the pulse combustor and ejector without compromising the device's performance. In fact, it is found that in several of the compact configurations analyzed, the system pressure gain actually increased.

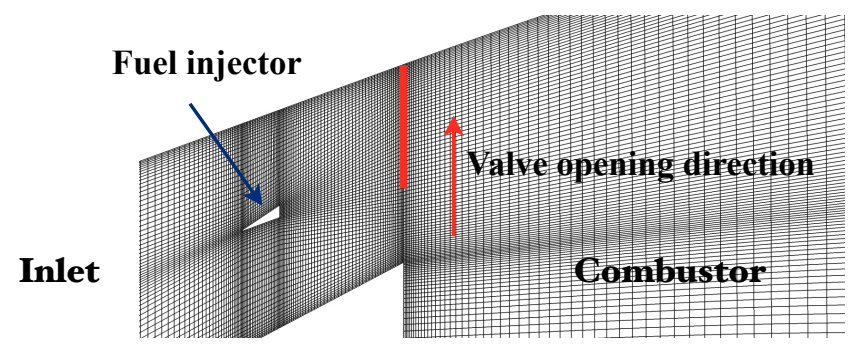

Figure 2. Vertically sliding valve model.

\section{Numerical Formulation}

The analysis is based on the axisymmetric, unsteady, Reynolds-averaged Navier-Stokes equations for a multispecies, thermally perfect, chemically reacting gas. They can be expressed in the following conservation form for a gas mixture containing $n$ species and in general curvilinear coordinates $(\xi, \eta)$

$$
\frac{\partial \mathbf{Q}}{\partial t}+\frac{\partial\left(\mathbf{F}-\mathbf{F}_{v}\right)}{\partial \xi}+\frac{\partial\left(\mathbf{G}-\mathbf{G}_{v}\right)}{\partial \eta}+j\left(\mathbf{S}-\mathbf{S}_{v}\right)=\mathbf{W}
$$

where

$$
\mathbf{Q}=J^{-1}\left[\rho_{1}, \rho_{2}, \ldots, \rho_{n}, \rho u, \rho v, e\right]^{T}
$$

is the vector of dependent variables. The equations describe two-dimensional flow $(j=0)$ or axisymmetric flow $(j=1)$. A detailed description of all the terms appearing in Eq. (1) and the additional state and constitutive equations needed to close the system are given in Ref. 10. The turbulence model used in the calculations is the Spalart-Allmaras oneequation model. ${ }^{11}$ Adiabatic walls are assumed for all cases analyzed.

The fuel considered in this study is gaseous Jet- $\mathrm{A}$, which is modeled as a $\mathrm{C}_{11} \mathrm{H}_{21}$ species. A reduced combustion mechanism, based on the model developed by Ajmani et al., ${ }^{12}$ consisting of 10 elementary reactions among 11 species was utilized. This mechanism has been successfully used in detonation and lean direct injection combustor systems studies. ${ }^{13-15}$

§ An alternative poppet valve model has also been developed in a previous study (see Ref.8). 
The numerical method used for solving the governing equations is described in detail in Refs. 10,16 and17 and briefly summarized here. The equation set is solved using a fully implicit, variable-step backward differentiation formula method. In this study, the temporally first-order backward Euler version of the scheme is used. The numerical fluxes are evaluated using a second-order spatially accurate total variation diminishing scheme. The resulting equations are then linearized in a conservative manner and solved iteratively using a lower-upper relaxation procedure consisting of successive Gauss-Seidel sweeps.

\section{Rayleigh Efficiency}

As previously stated, RPCs are unsteady thermo-acoustic devices in which heat released by combustion is coupled with the acoustic waves. It is possible to give a more precise mathematical description of this coupling and quantify the conditions under which heat release encourages fluctuations in the flow field, which is a desirable feature in pulse combustors since it is the mechanism by which pressure gain is attained.

Starting from the Euler equations with heat addition, simplified under the assumption of small disturbances, the following equation for the acoustic energy can be derived ${ }^{18,19}$

$$
\frac{\partial}{\partial t}\left[\frac{1}{2} \frac{p^{\prime}}{\rho_{r e f} c_{r e f}^{2}}+\frac{1}{2} \rho_{r e f} u^{\prime} \cdot u^{\prime}\right]+\nabla \cdot\left(p^{\prime} u^{\prime}\right)=\frac{\gamma-1}{\gamma p_{r e f}} p^{\prime} Q^{\prime}
$$

where $u^{\prime}, p^{\prime}$ and $\mathrm{Q}^{\prime}$ are small disturbances in velocity, pressure and heat release respectively, and the subscript ref indicates reference quantities. The two terms in the square brackets are a potential energy term (first term) and the kinetic energy (second term), and the divergence term on the left side corresponds to the flux of acoustic energy. The source term on the right hand side represents the production of acoustic energy. Integrated over a volume V (with boundary $\mathrm{S}$ ) and over a period of oscillation, $T_{c}$, gives

$$
\frac{\gamma-1}{\gamma p_{\text {ref }}} \int_{T_{c}} \int_{V} p^{\prime} Q^{\prime} d V d t=\int_{T_{c}} \int_{S} p^{\prime} u^{\prime} \cdot n d S d t
$$

This equation provides a quantification of the coupling between the pressure fluctuations, $p^{\prime}$, and the heat release, $Q^{\prime}$ necessary to maintain the acoustic disturbances, and is known as Rayleigh's criterion. The left hand term is known as the Rayleigh efficiency. Unlike most efficiency definitions, the Rayleigh efficiency is not bounded between zero and one but is, in fact, unbounded and can even be negative (although a flow field with negative Rayleigh efficiency would not be able to maintain the pressure disturbances for a significant amount of time). In the present finite rate formulation the Rayleigh efficiency is calculated as follows.

The heat release per unit volume is given by

$$
q=-\sum_{1}^{n} \rho_{i} h_{i}^{0}
$$

Referring to Fig. 3, a control volume is considered in the combustor. This control volume should be large enough to contain all the fuel that has been injected into the combustor.

The total heat release at a given time is given by

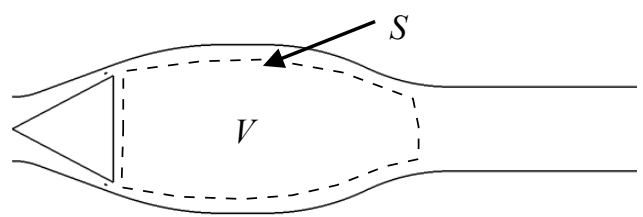

$$
Q_{v}(t)=\int_{V} q(t) d V
$$

and the heat release flux is given by

$$
\dot{Q}_{S}(t)=\int_{S} q(t) \vec{v} \cdot \vec{n} d S
$$

Figure 3. Control volume used in computation of Rayleigh efficiency.

The instantaneous rate of heat release is then given by

$$
\dot{Q}(t)=\frac{\partial Q_{V}(t)}{\partial t}+\dot{Q}_{S}(t)
$$

and the time-averaged rate of heat release over one RPC cycle is

$$
\overline{\dot{Q}}=\int_{T_{c}} \frac{\dot{Q}(t) d t}{T_{c}}
$$

The instantaneous average combustor pressure in the control volume is given by

$$
p_{\text {avg }}(t)=\int_{V} \frac{p(t) d V}{V}
$$


and the time-averaged combustor pressure is

$$
\bar{p}_{\text {avg }}=\int_{T_{c}} \frac{p_{\text {avg }}(t) d t}{T_{c}}
$$

The Rayleigh efficiency is then computed by the following expression

$$
\eta_{R}=\frac{\int_{T_{c}}(\dot{Q}(t)-\bar{Q})\left(p_{\text {avg }}(t)-\bar{p}_{\text {avg }}\right) d t}{\overline{\dot{Q}} \bar{p}_{\text {avg }} T_{c}}
$$

where the constant ahead of the integral in Eq.(4) has been ignored in the definition of the Rayleigh efficiency, since in the present numerical formulation $\gamma$ is not constant (and is not used, except at certain boundary conditions).

The definition of the Rayleigh efficiency given in Eq. (12) is similar to that used by Keller et al, ${ }^{3}$ with the exception of a constant ahead of the integral. However, it should be mentioned that Blackburn and Miller ${ }^{20}$ have recently introduced an alternative definition of the Rayleigh efficiency (based on the concept of increased potential to do shaft work) to analyze the performance of pulse combustors.

The Reynolds efficiency given in Eq. 12 has been computed for several configurations and is presented in the next section as an additional figure of merit that characterizes the performance of EERPCs.

\section{Numerical Simulations}

As mentioned in the introduction, the EERPC configurations considered in previous studies ${ }^{4-8}$ were relatively long, making them difficult to integrate into gas turbine engines. One of the objectives of the present study is to investigate the performance of more compact configurations with respect to both the pulse combustor and ejector components. The focus is on the system pressure gain, defined here as the difference between the mass-averaged total pressure at the ejector exit and the inflow total pressure (expressed as a percentage of the inlet total pressure ${ }^{* *}$.

The performance of EERPCs is investigated at inflow conditions of $p_{0}=10$ bar and $\mathrm{T}_{0}=550 \mathrm{~K}$, which may be considered as representative conditions for the exit of a modern turbofan compressor at cruise conditions.

Figure 4 shows the different configurations that were analyzed. The baseline configuration is shown in Fig. $4 \mathrm{a}$, and is the same one considered in Ref. 8. The others represent more compact configurations in which the RPC and/or the ejector have been shortened.

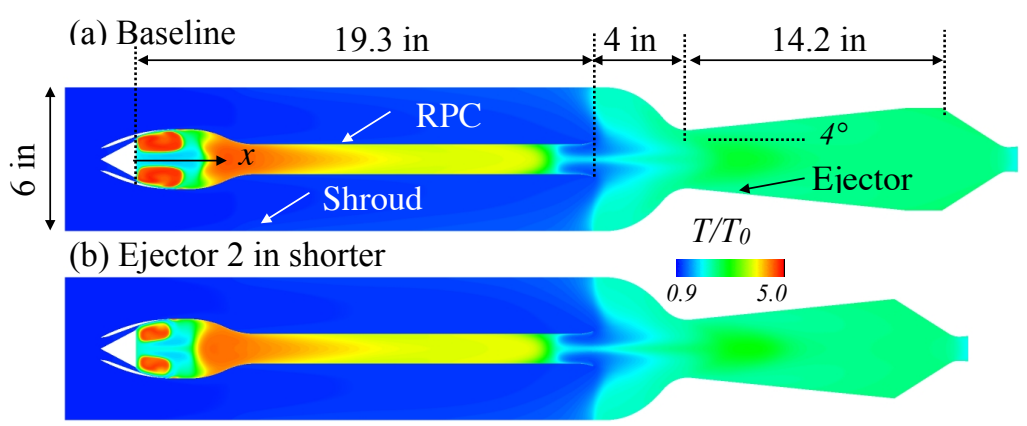

(c) Ejector 4 in shorter

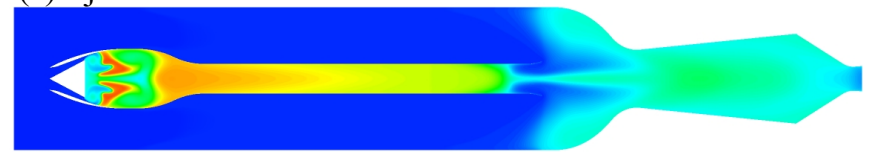

(d) Ejector and pulse combustor 2 in shorter

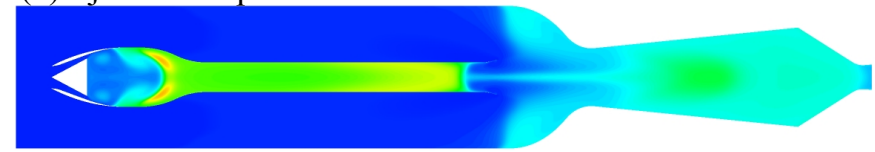

(e) Ejector 2 in shorter, pulse combustor 4 in shorter

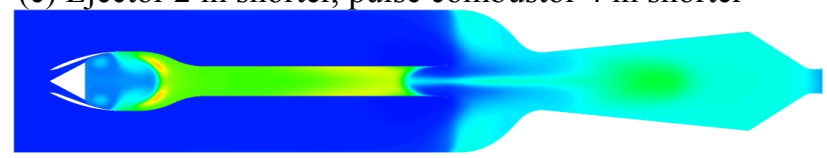

(f) Ejector and pulse combustor 4 in shorter

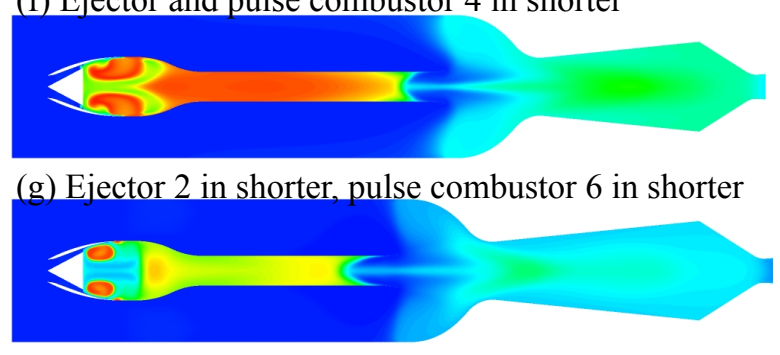

\section{Figure 4. EERPC configurations (showing temperature contours).}

** The mass-averaged total pressure has been shown to be inappropriate for pressure-gain combustion concepts with large variations in properties over the course of a cycle; however, it is convenient and acceptable in this study, in which variations at the ejector exit are quite small. 


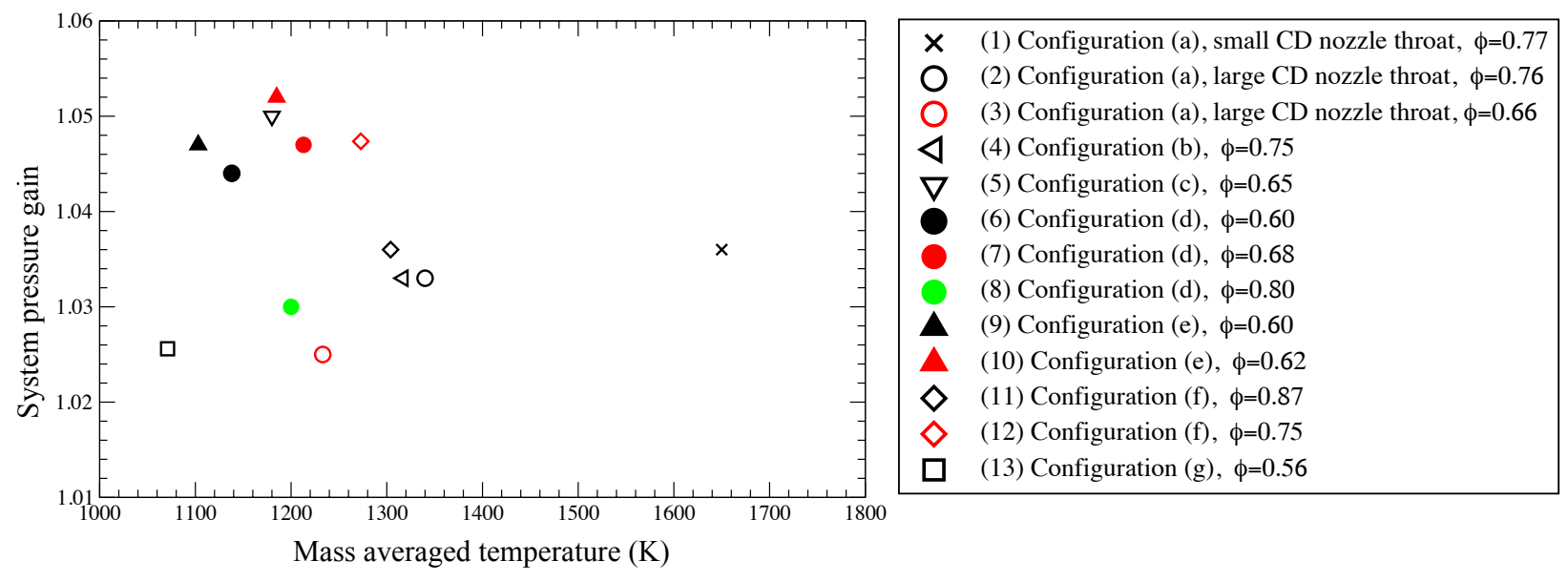

Figure 5. System pressure gain for various EERPC configurations.

Figure 5 summarizes the system pressure gain plotted as a function of mass-averaged exit temperature for thirteen EERPC configurations. One restriction placed on the current (and previous) studies is that the maximum temperature allowed by a turbine should not be exceeded. Typically, a temperature ratio of 2 across the combustor is encountered in aircraft gas turbine engines. Therefore, in these studies, the mass-averaged temperature at the exit of the shrouded EERPC is sought that is approximately $1100 \mathrm{~K}$. The results presented in Fig. 5 show that with one exception, all cases are within approximately $20 \%$ of the target temperature. The one exception is case \#1, which consists of a configuration with a smaller CD nozzle throat diameter (0.86 in. vs $1.02 \mathrm{in}$.). The smaller throat restricted the bypass airflow too much resulting in high exit temperatures. All other configurations in Fig. 5 utilized the larger CD nozzle throat.

This figure shows that the pressure gain tends to fall within two clearly defined regimes depending primarily on the particular configuration and equivalence ratio. All six cases belonging to the high performance group have three common features: a compact configuration, a period doubling behavior (described later), and an RPC equivalence ratio in the range $0.6<\phi<0.75$. Note that the pressure gain achieved with some of these compact configurations can exceed 5\%, a level significantly higher than that obtained in previous studies. ${ }^{6-8}$ Previous analyses have shown that these levels of pressure gain should result in substantial reductions in specific fuel consumption ${ }^{7}$ (for the same operating conditions).

Table A1, in the appendix, provides additional information on the 13 cases. In particular, it is noted that the average operating frequency (the frequency can vary from cycle to cycle in some cases) is primarily a function of the equivalence ratio, $\phi$, and the length of the RPC. Higher values of $\phi$ result in higher temperatures and therefore higher sound speeds. The higher temperatures also reduce the ignition delay time. Both of these factors contribute to an increase in operating frequency. Shorter RPC lengths reduce the distance the gas dynamic waves need to travel, resulting in higher operating frequency. The bypass ratio is within a narrow range between $1.6<\beta<2.0$ for most cases. The exceptions are case \#1 which, as previously mentioned, utilizes a smaller CD nozzle throat that constricts the airflow, and case \#13, whose small RPC length led to poor synchronization resulting in diminished primary airflow.

An in-depth analysis of several of the cases shown in Fig. 5 was carried out with the goal of understanding the factors that determine pressure gain in EERPCs. This analysis is presented below.

Figure 6 shows temperature contours (top half of each figure) and fuel mass fraction contours (bottom half) at various times during one cycle for the baseline configuration (case \#2). A detailed description of the flow and combustion processes taking place in the RPC is given in previous papers. ${ }^{4-8}$ Here only a brief description is given.

Figure 6a shows the flowfield at the start of the cycle. Combustion is seen occurring mainly at the center of the primary vortex. Subsequent figures show the blowdown process and the opening of the inlet valve (Fig. 6b), allowing air to flow into the pulse combustor and forming a strong toroidal vortex (Figs. 6c-6d). Fuel injection starts at $t=2.12 \mathrm{~ms}$ and ends at $t=2.62 \mathrm{~ms}$. Ignition in the vortex core is observed in Fig. $6 \mathrm{e}$. The valve is fully closed at $t=$ $2.79 \mathrm{~ms}$ (Fig. 6f) and the cycle repeats. Although the flow is unsteady, the CD nozzle remains choked throughout the entire cycle (this is also true for all the other cases in Fig. 5). The frequency of operation is $359 \mathrm{~Hz}$, and the 
equivalence ratio is $\phi=0.76$. The combustion process shown in Fig.6, in which all combustion occurs in the combustion chamber and none in the tailpipe, is consistent with recent experimental observations reported by Anand et. al. ${ }^{21}$

The results for case \#2 show that most of the fuel goes into the primary vortex and combustion starts at the vortex core which subsequently spreads outwards through the combustion chamber. This fuel distribution and combustion process was shown to be optimal for a pulse combustor operating by itself ${ }^{7}$ (i.e., without the ejector, shroud and $\mathrm{CD}$ nozzle). Here, when combined with an ejector and shroud, it does not produce the highest system pressure gain due to the complex interactions that exist between the RPC and the ejector/shroud.

Figure 7 shows the pressure history at the head-end of the combustor for 10 cycles. Alternating colors are used in this and subsequent figures to differentiate each cycle. The pressure profile is nearly uniform for every cycle, although some variation exists in the peak pressures. Figure 8 shows the rate of heat release and the average combustor pressure for 4 cycles. Indicated in Fig. 8 are the time-averaged combustor pressure ratio

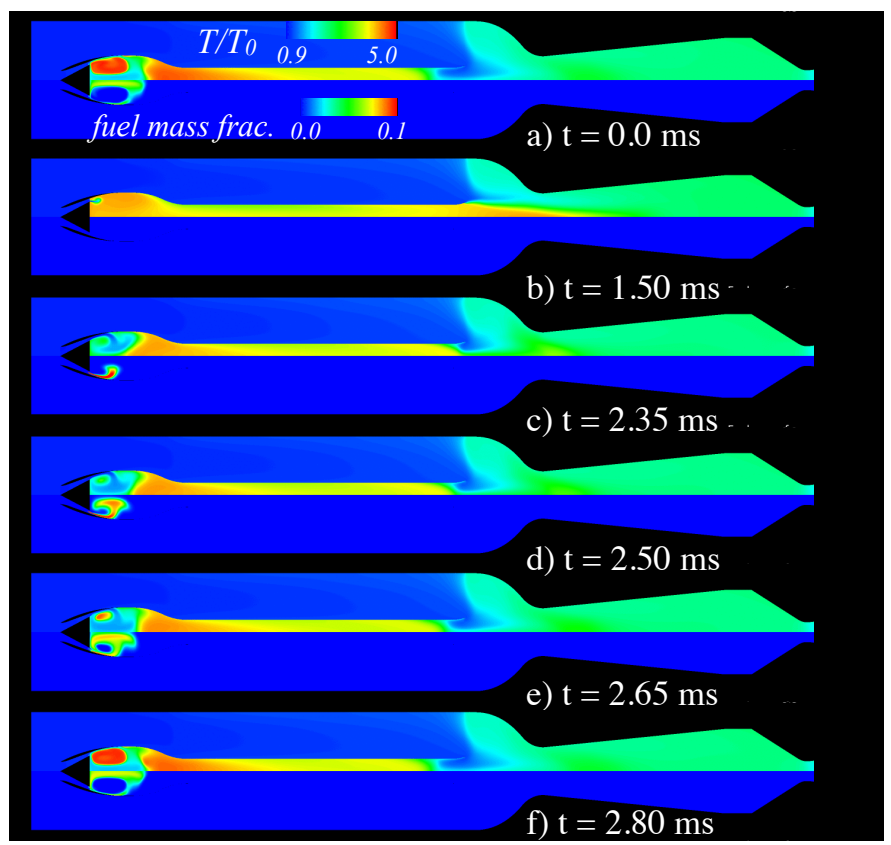

Figure 6. Temperature contours (top half) and fuel mass fraction contours (bottom half) for the baseline EERPC configuration during one cycle (case \#2). and the Rayleigh efficiency for each cycle. On average, higher values of the Rayleigh efficiency correspond with higher average combustor pressures.

Figure 9 illustrates the typical process required to reach a limit cycle in the EERPC simulations. This figure shows mass-averaged temperature computed at the end of the diverging section of the EERPC $(x=37.5$ in. for the baseline case, see Fig. 4a). This calculation was started from a previously obtained solution. After approximately 18 cycles, the mass-averaged temperature stabilized at approximately $1330 \mathrm{~K}$. Figure 10 shows the mass-averaged total pressure computed at the end of the diverging section of the EERPC, and represents the system pressure gain, as defined previously. For this case, the system pressure gain converges to a value of around $3.4 \%$.

Figure 11 shows an $x-t$ diagram of pressure along the pulse combustor centerline (Fig. 11a) and along a path through the middle of the shroud/ejector (Fig. 11b) during 2 cycles of the baseline EERPC. A detailed description of the wave dynamics in pulse combustors has been presented before in Ref. 7. Here we only highlight some of the important features of this case. This plot confirms the results presented in Fig. 8 in that the combustion process occurs rapidly, and in a very small region in the combustor (specifically, the vortex core). This combustion process

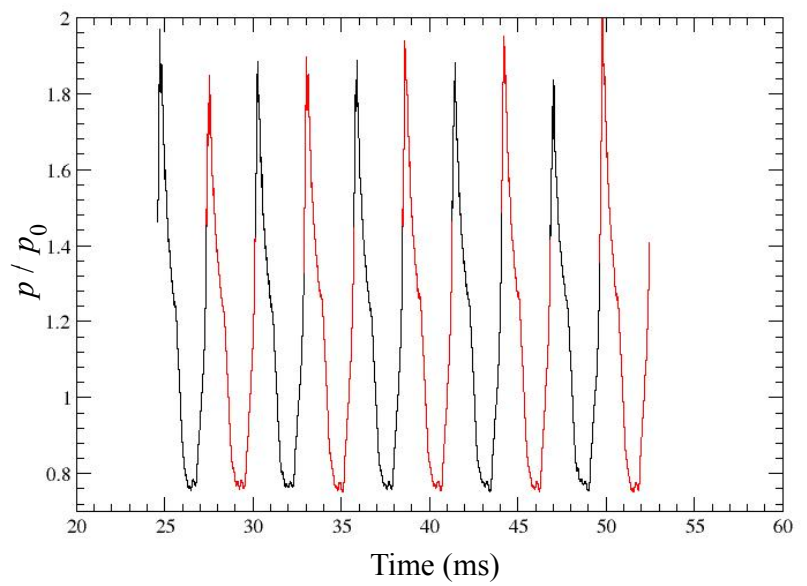

Figure 7. Combustor head-end pressure ratio for baseline configuration (case \#2).

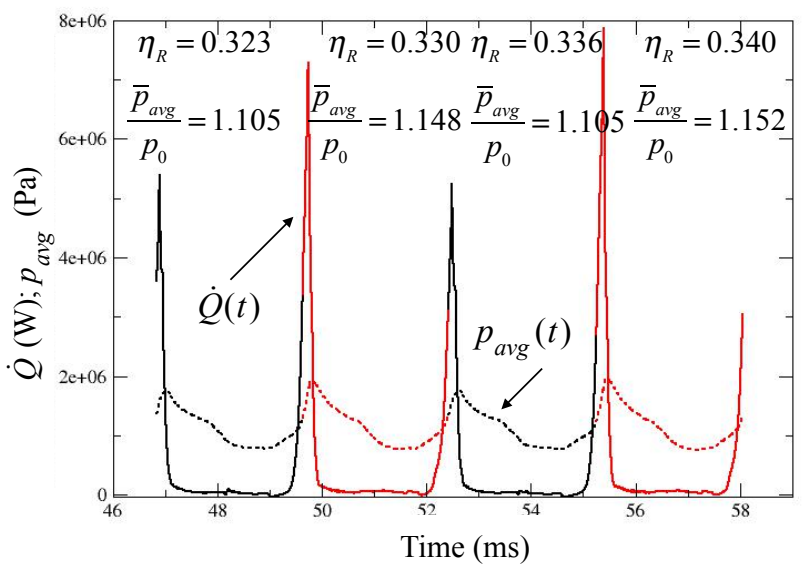

Figure 8. Rate of heat release and average combustor pressure for case \#2. 


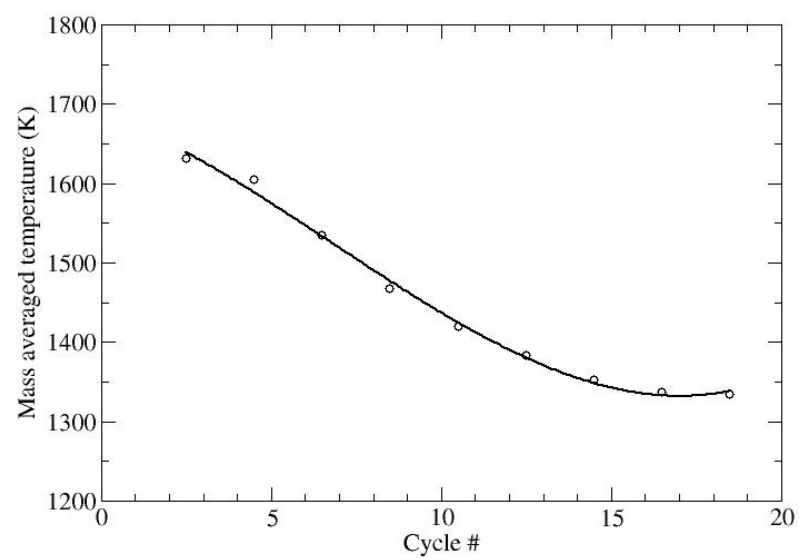

Figure 9. Mass-averaged temperature at the exit of the EERPC (case \#2).

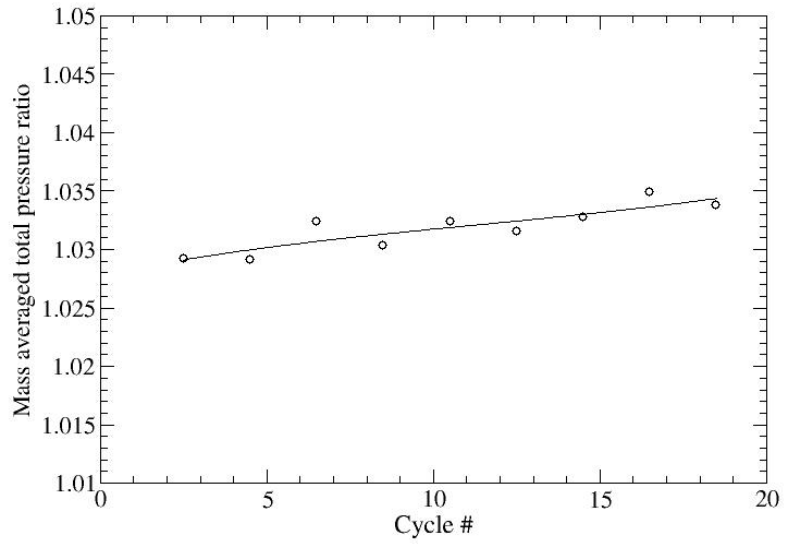

Figure 10. Mass-averaged total pressure ratio at the exit of the EERPC (case \#2).

generates a strong compression wave (reaction shock) that propagates downstream. When this compression wave reaches the end of the RPC, it is reflected as an expansion wave. Some important events related to the valve and fuel injection timing are indicated in this figure. The two cycles are very similar, although somewhat more intense combustion and a stronger reaction shock is observed for cycle 2. The $\mathrm{x}$-t diagram for the bypass air (Fig. 11b) shows a classic acoustic wave pattern. The strong waves propagating upstream and downstream near the ejector throat are caused by the interaction between the combustion products exiting the RPC and the ejector wall.

The Rayleigh efficiency was also computed for case \#3, which also involves the baseline configuration but at a lower equivalence ratio. It produced a significantly lower pressure gain. The pressure history for this case is shown
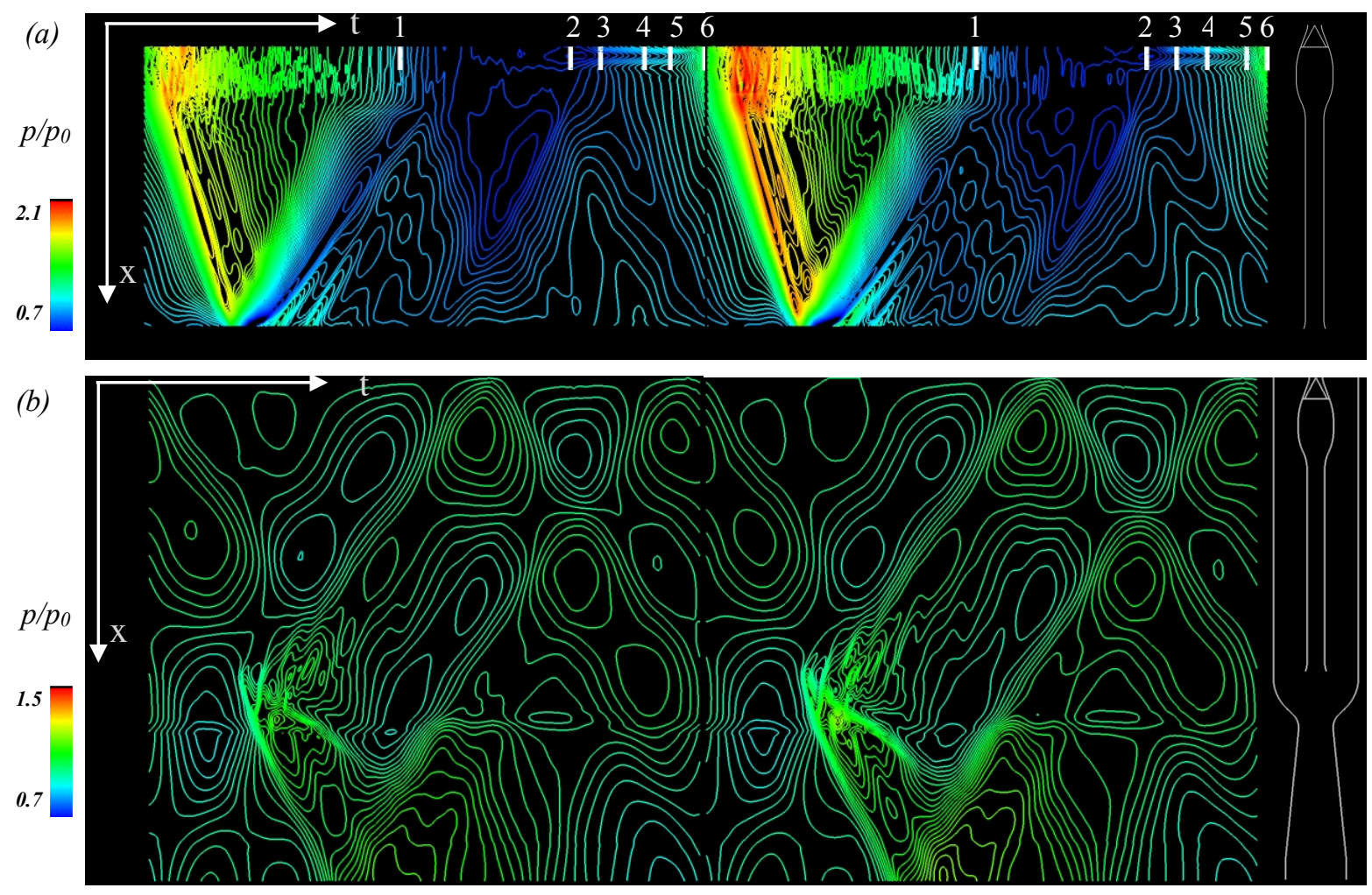

Figure 11. x-t pressure diagram (case \#2); (a) pulsejet centerline; (b) Bypass air. Time labels: 1-valve opening start, 2-fuel injection start, 3-valve fully open, 4-fuel injection end, 5 -valve closing start, 6 -valve fully closed. 


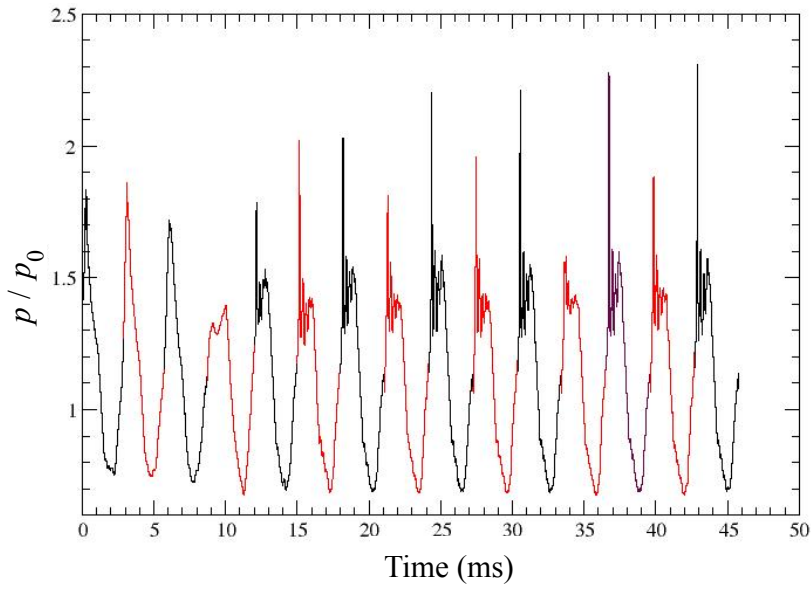

Figure 12. Combustor head-end pressure ratio for baseline configuration (case \#3).

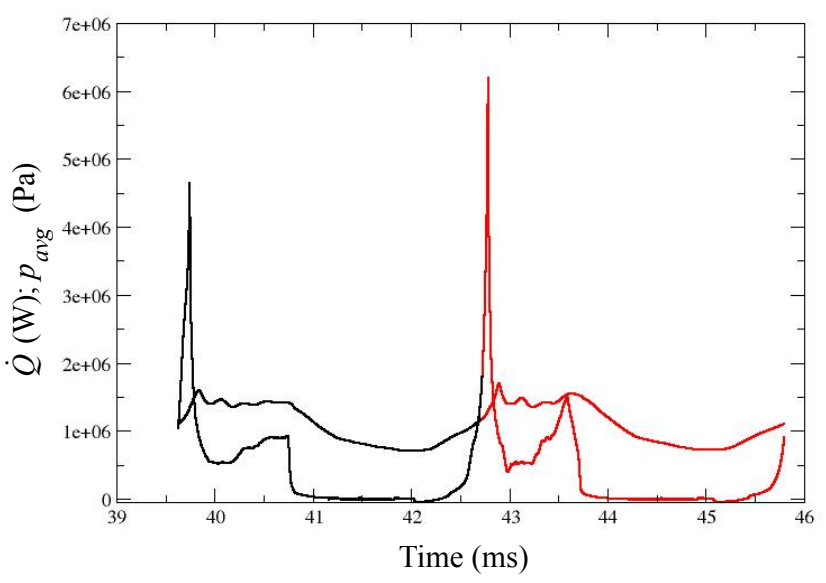

Figure 13. Rate of heat release and average combustor pressure for case \#3.

in Fig. 12. Note that the pressure history now exhibits a period-doubling behavior; that is, the flow is periodic but repeats itself every other cycle. In comparison, the pressure profile for case \#2 shows a nearly uniform profile for every cycle (Fig. 7). Such occurrences of period doubling, and even period tripling were observed also in our previous studies, ${ }^{6,7}$ and have been observed also in connection with pulse detonation rocket-based combined cycle propulsion concepts 22 .

The rate of heat release and the average combustor pressure are shown in Fig. 13. Note that the rate of heat release shows a sharp peak followed by a smaller and flatter secondary heat release period. The rate of heat release is noticeable different for the two cycles plotted. The average combustor pressure shows a much fatter profile compared with that in Fig. 8. Both the time-averaged combustor pressure ratio and the Rayleigh efficiency are lower than case \#2. The mass-averaged temperature and the pressure gain are shown in Figs. 14 and 15. The temperature decreased as a result of the lower equivalence ratio in the RPC, and the pressure gain stabilized at around $2.5 \%$, significantly lower than that obtained for case \#2.

The highest pressure gain in the present study was obtained for case \#10 which was based on the compact configuration shown in Fig. 4e. The pressure history for this case is shown in Fig. 16. As in case \#3 discussed previously, this case also exhibited a period doubling mode, with larger differences between cycles but with more uniform pressure profiles. The repeatability of the pressure profiles over successive cycles is very close.

The rate of heat release and the average combustor pressure are shown in Fig. 17. The difference in heat release between the two cycles is very large in this case, and the heat release occurs in two steps as in case \#3. This is illustrated in Fig. 18 which shows temperature contours and fuel mass fraction contours at various times during two cycles.

Figure 18a shows the flowfield at the start of the first cycle. Combustion starts at the outer edge of the secondary vortex creating the first peak in the rate of heat release seen in Fig. 17. A short time afterwards, a very intense

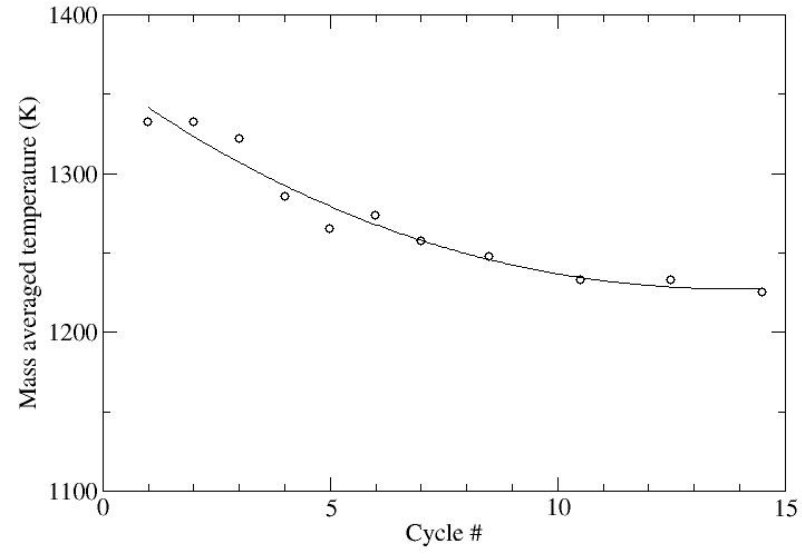

Figure 14. Mass-averaged temperature at the exit of the EERPC (case \#3).

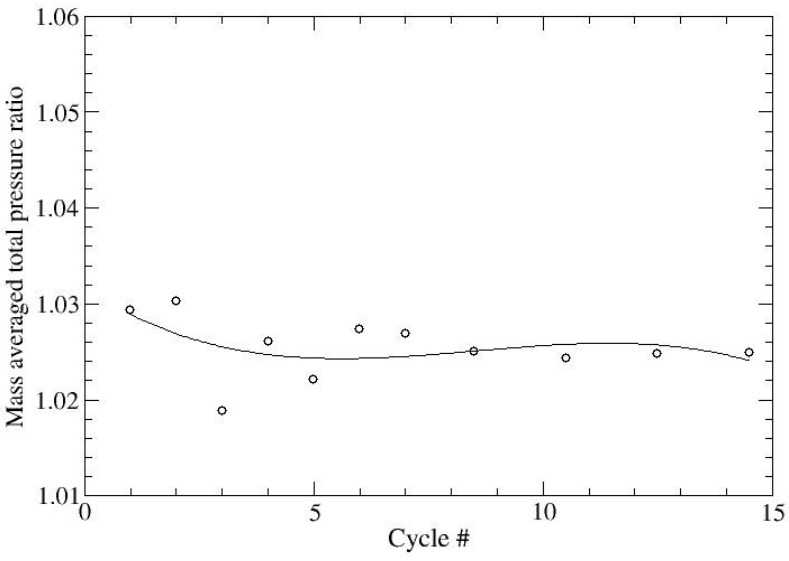

Figure 15. Mass-averaged total pressure ratio at the exit of the EERPC (case \#3). 


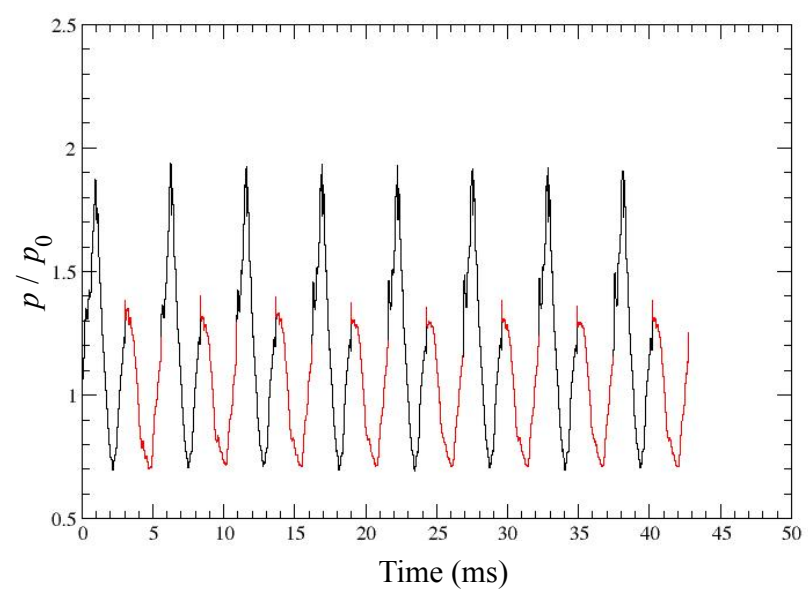

Figure 16. Combustor head-end pressure ratio (case \#10).

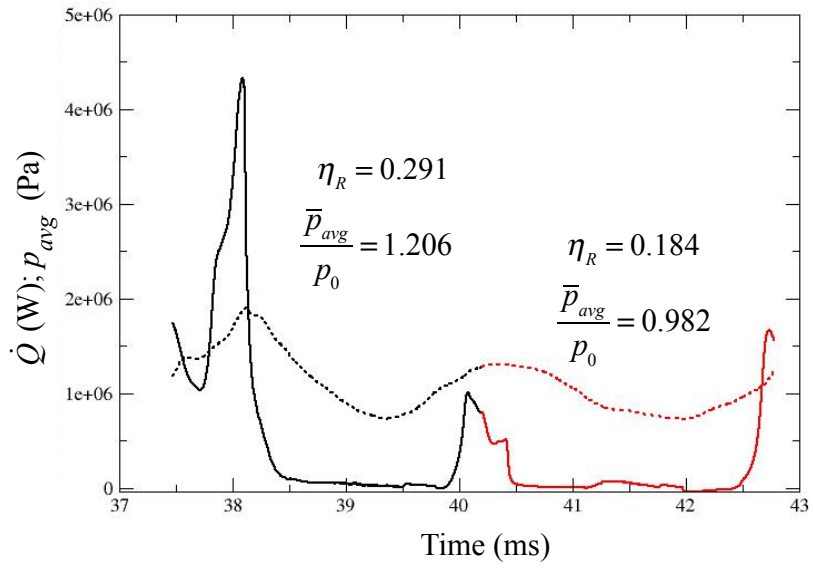

Figure 17. Rate of heat release and average combustor pressure (case \#10).

second combustion front is seen starting at the core of the primary vortex (Figs. 18b and 18c) which creates the second, larger peak in the rate of heat release. It is interesting to note that Keller et. al. ${ }^{23}$ observed the same combustion process (i.e., ignition at the outer edge of the vortex and subsequent propagation into its center) in their experimental studies of valved pulse combustors. Offord et. al. ${ }^{9}$, also reported that ignition occurred at the outer edge of the vortex in their experimental study of a valveless pulse combustor.

Subsequent figures show the blowdown process and the opening of the inlet valve (Fig. 18d). Fuel injection starts at $t=2.29 \mathrm{~ms}$, but the overall amount of fuel injected during this cycle is very small and the combustion is very week (Figs. 18e-18g). The valve is fully closed at $t=2.74 \mathrm{~ms}$, marking the end of this cycle. The blowdown process continues and the valve opens again at $t=3.64 \mathrm{~ms}$ and fuel injection starts at $t=4.64 \mathrm{~ms}$ and ends at $t=4.99 \mathrm{~ms}$. For this second cycle, the amount of fuel injected is much larger. At the end of this cycle (Fig. 18n) the flow has returned to its initial state (i.e., Figures $18 \mathrm{n}$ and $18 \mathrm{a}$ are nearly identical) and the cycle repeats.

An x-t pressure diagram of the two cycles is shown in Fig. 19, and provides additional insight into the flow and combustion processes taking place. During cycle 1 , it is observed that when the fuel injection starts at time t 2 the
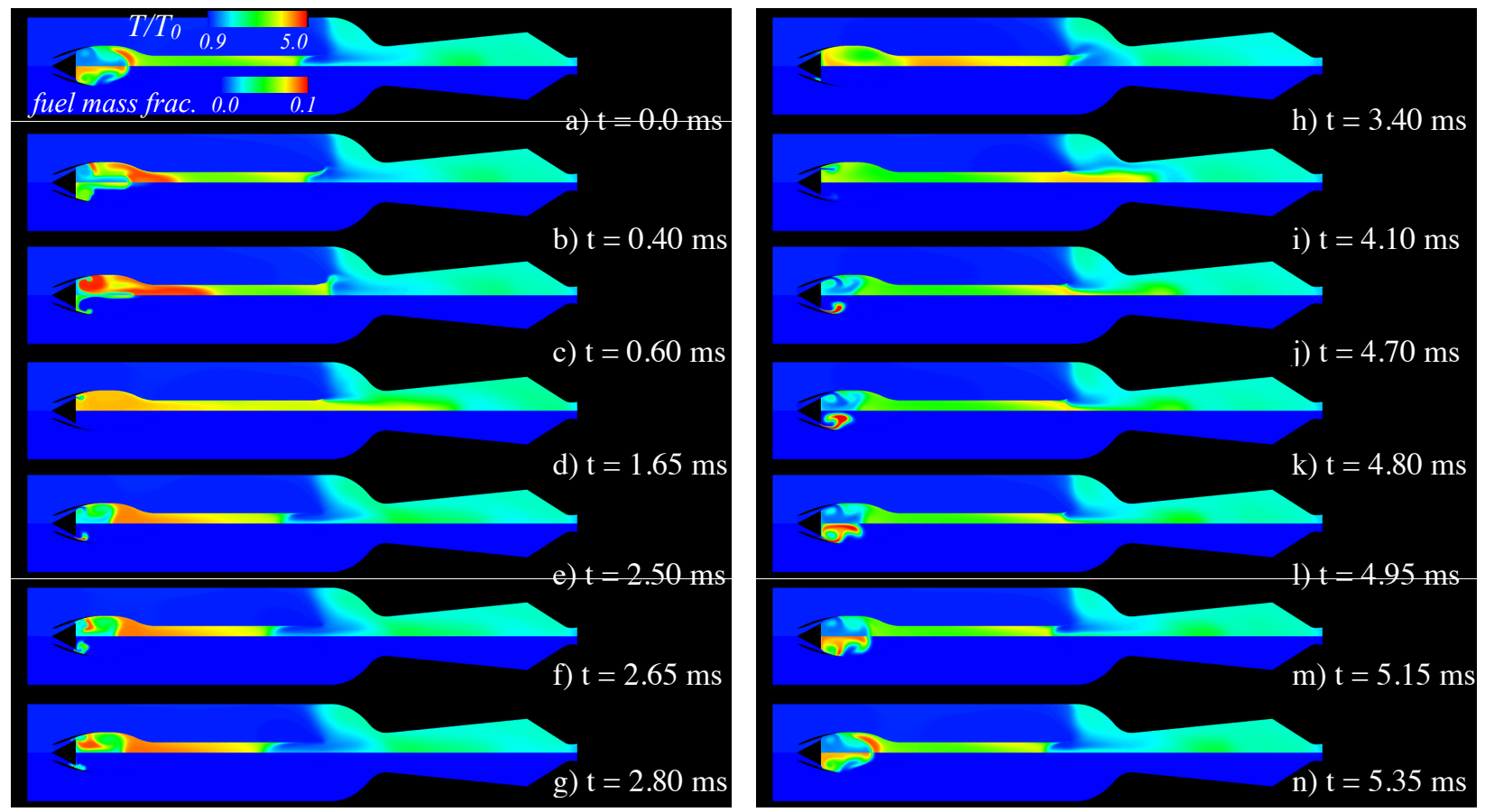

Figure 18. Temperature contours (top half) and fuel mass fraction contours (bottom half) during two cycles for case \#10.

American Institute of Aeronautics and Astronautics 

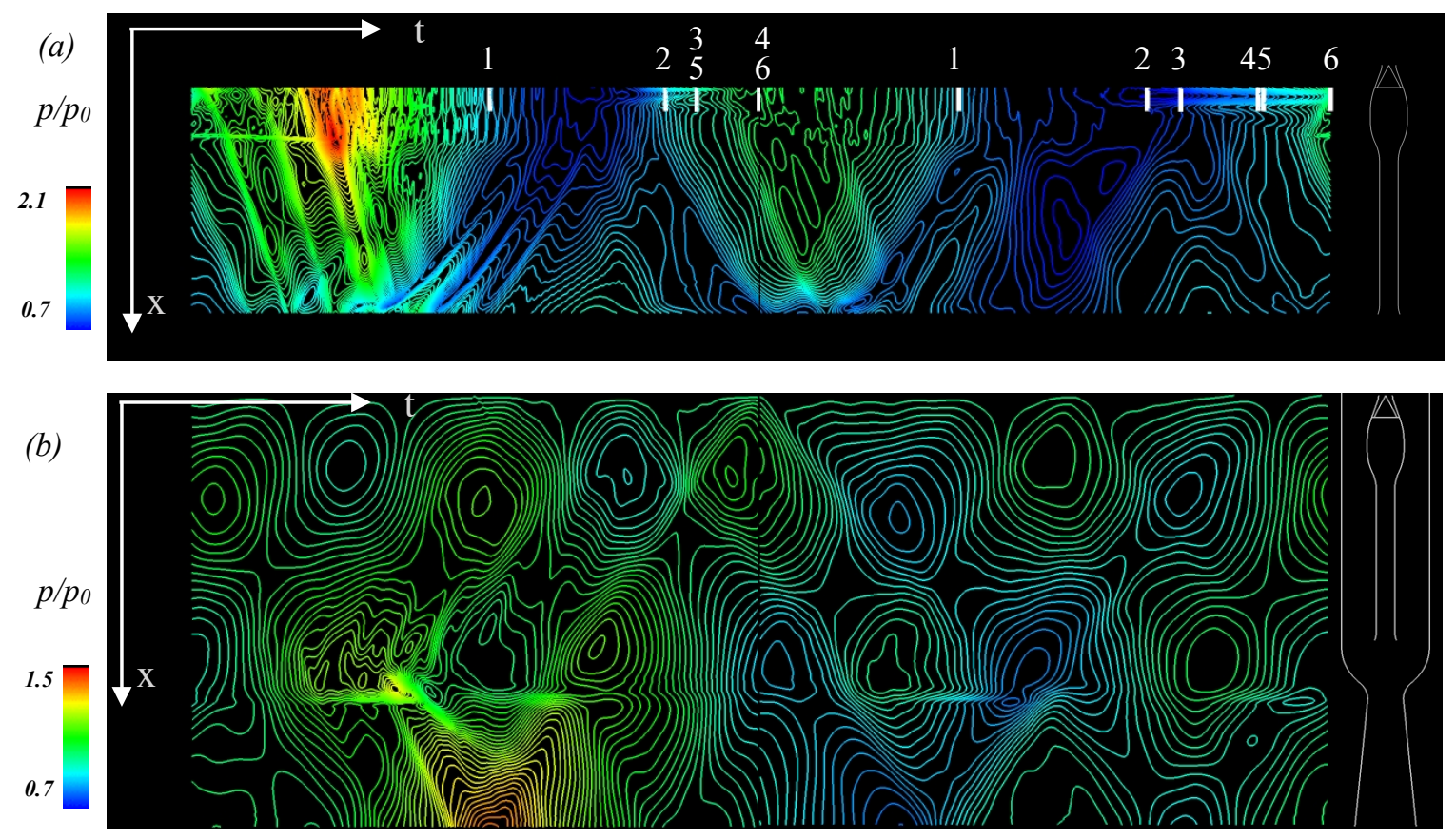

Figure 19. x-t pressure diagram (case \#10); (a) pulsejet centerline; (b) Bypass air. Time labels: 1-valve opening start, 2-fuel injection start, 3-valve fully open, 4-fuel injection end, 5-valve closing start, 6-valve fully closed.

pressure at the head-end of the combustor is already quite high (this can be seen by comparing the pressure levels at time $\mathrm{t} 2$ between cycle 1 and cycle 2). By the time the valve is fully open at $\mathrm{t} 3$, the pressure is high enough that the valve begins to close immediately (time t5) under the current computational model. The fuel injection is occurring against a high combustor pressure that continues to increase from $\mathrm{t} 2$ until t 6 when the valve is fully closed. The end of fuel injection, $t 4$, nominally extends until the valve closes, however, fuel injection effectively ends sooner, when the pressure in the combustor is higher than the fuel injection pressure. This occurs sometime between times 5 and t6. This is the reason that during this cycle, the amount of fuel injected and the resulting heat release are so low. The second cycle, on the other hand operates normally as in case \#2 (Fig. 11a).

It is interesting to note in the x-t diagram along the ejector path (Fig. 19b) the high pressure region near the ejector exit during cycle 1, which is consistent with the high pressure gain obtained in this case. It is somewhat surprising that this case, for which one of the two cycles is so poorly synchronized, produces the highest system pressure gain (5.2\%), and is an indication of the strong and complex flow interaction involving the RPC, ejector and shroud components.

The mass-averaged temperature and the pressure gain for this case are shown in Figs. 20 and 21. Each data point in these figures consists of the average of two cycles.

Finally, results are presented for cases \#11 and \#12, which show a significant increase in pressure gain following a relatively small reduction in equivalence ratio from $\phi=0.87$ to $\phi=0.75$. Figures 22 and 23 show the pressure history at the head-end of the combustor and the rate of heat release (and average combustor pressure) for case \#11 during 2 cycles. This case operates in single period mode, although there is a small variation in peak pressure and average combustor pressure from cycle to cycle. The Rayleigh efficiency is nearly constant at $\eta_{R} \approx 0.2$. A reduction in equivalence ratio causes the EERPC to switch to a period doubling mode, as shown in Fig. 24. In this case, there is also a low frequency mode superimposed on the fundamental RPC frequency. The different heat release profiles in each pair of cycles is shown in Fig. 25 which also indicates the values of the Rayleigh efficiency, which are both higher that those obtained in case \#11. Thus, as in the baseline case, higher values of the Rayleigh efficiency correspond to higher levels of pressure gain (for the same configuration). However, the results obtained in this study indicate that the Rayleigh efficiency cannot be used when comparing different configurations. For example, the Rayleigh efficiency for case \#2 utilizing the baseline configuration was $\eta_{R} \approx 0.34$ and the pressure gain was $3.4 \%$. The highest value of the Rayleigh efficiency for case \#10 was $\eta_{R}=0.29$ but the pressure gain was $5.2 \%$. 


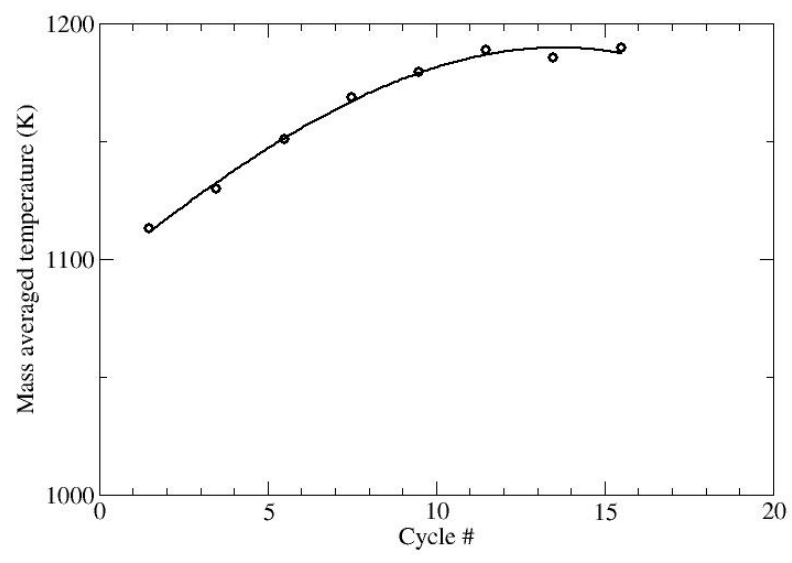

Figure 20. Mass-averaged temperature at the exit of the EERPC (case \#10).

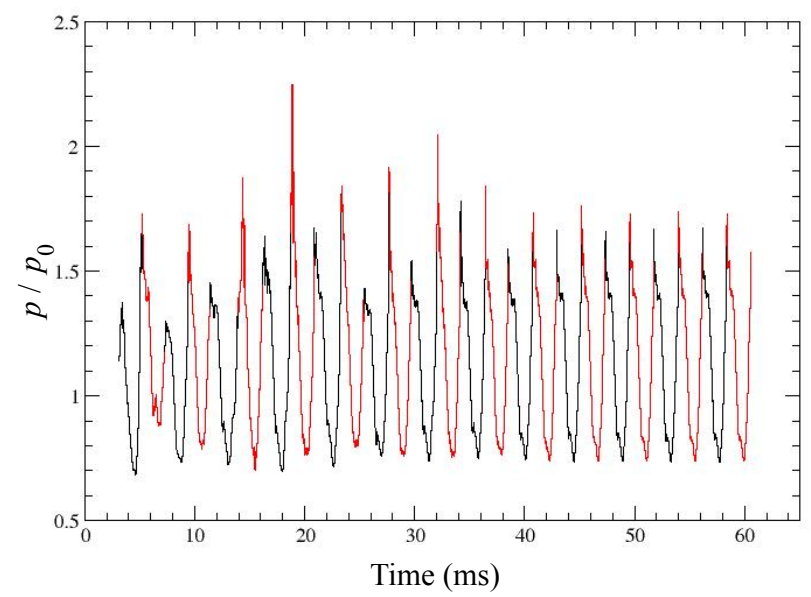

Figure 22. Combustor head-end pressure ratio (case \#11).

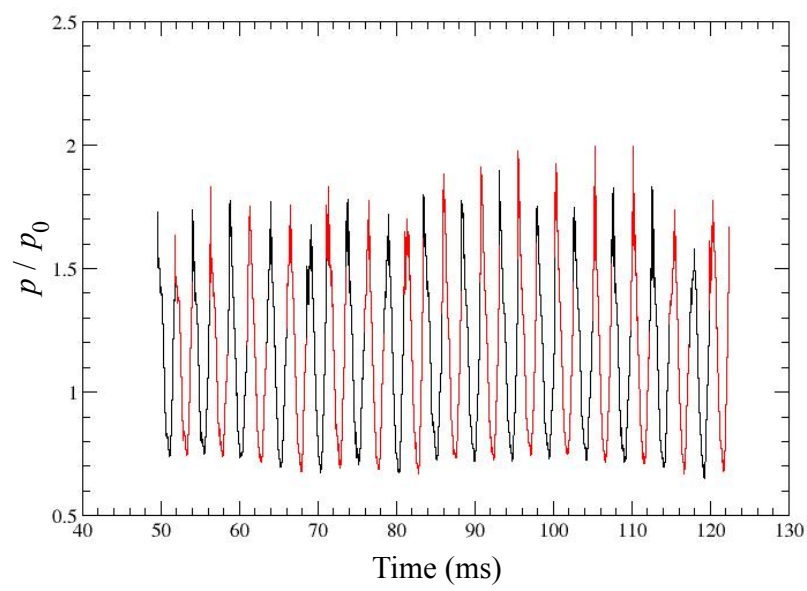

Figure 24. Combustor head-end pressure ratio (case \#12).

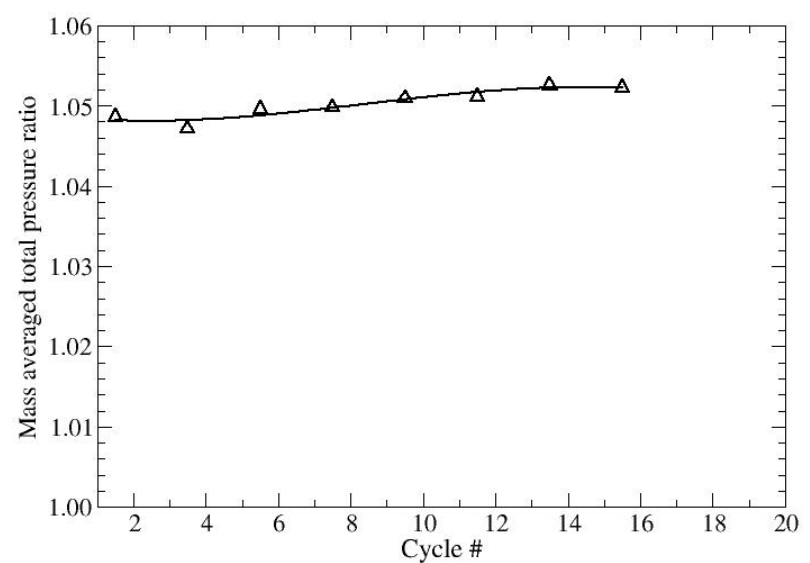

Figure 21. Mass-averaged total pressure ratio at the exit of the EERPC (case \#10).

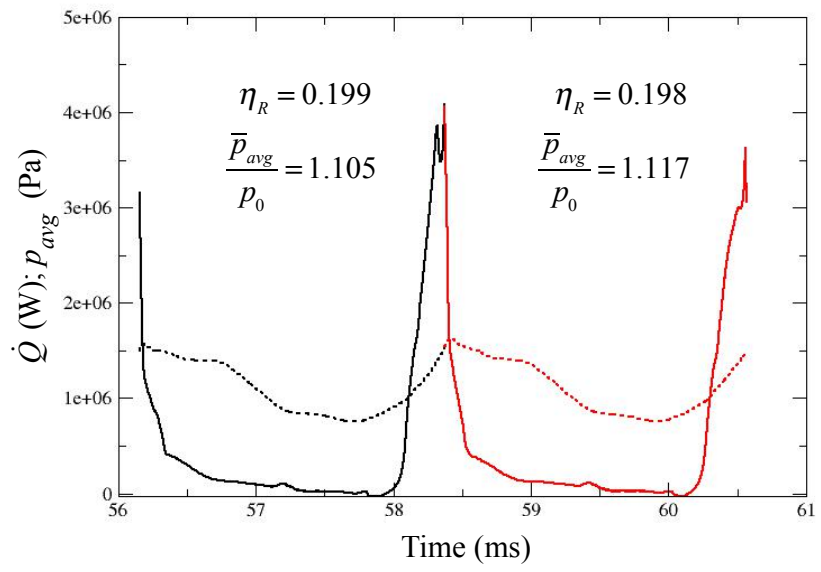

Figure 23. Rate of heat release and average combustor pressure (case \#11).

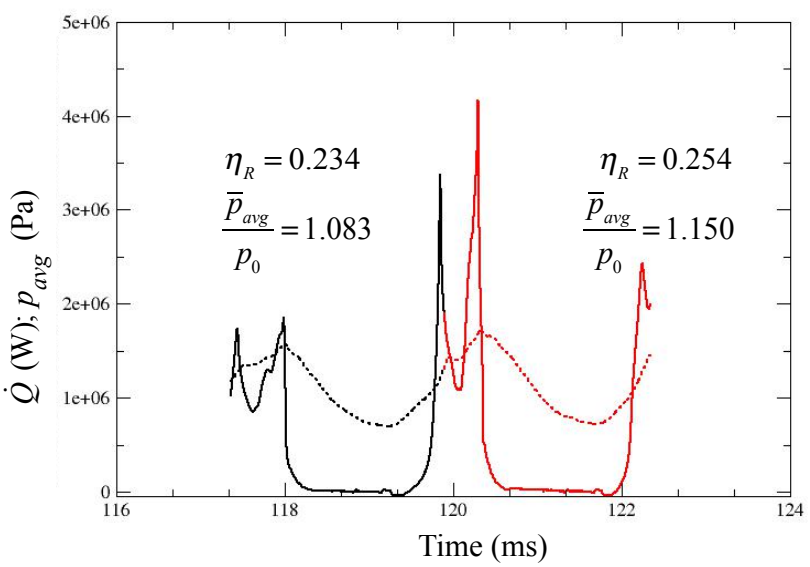

Figure 25. Rate of heat release and average combustor pressure (case \#12).

American Institute of Aeronautics and Astronautics 
One final comment is made regarding case \#13. This case utilized the shortest RPC (see Fig. 4g), and resulted in a very low pressure gain $(2.56 \%)$. In this case the RPC was so short that the gasdynamic waves, valve dynamics, fuel injection and heat release became severely out of synchronization, resulting in poor performance. The use of faster valves and adjustments to the fuel injection timing could improve the performance of this configuration.

\section{Conclusions}

One of the main objectives of this study was to investigate the performance of more compact EERPC configurations in which the pulse combustor and/or the ejector components were shortened. The focus was on the effects of these geometric changes on system pressure gain. Thirteen cases were analyzed, and the results showed that the pressure gain fell within two clearly defined regimes depending primarily on the particular configuration and equivalence ratio. All six cases belonging to the high-performance group had three features in common: a compact configuration, a period doubling behavior, and an RPC equivalence ratio in the range $0.6<\phi<0.75$. The pressure gain achieved with some of these high-performance configurations exceeded $5 \%$, a level significantly higher than that obtained in previous studies.

The Rayleigh efficiency was computed for several configurations and proved to be an important and relevant figure of merit for characterizing pulse combustor flowfields. The Rayleigh efficiency was seen to correlate with both average combustor pressure and system pressure gain when the same configuration was being analyzed, however, it should not be used to compare different configurations.

The EERPC configurations that produced the highest system pressure gain had flow and combustion characteristics that would be suboptimal for an RPC operating by itself (i.e., without the ejector and shroud). This is an indication of a strong and complex flow interaction involving the RPC, ejector and shroud components that, while still not completely understood, can potentially be exploited to increase performance even more.

\section{Acknowledgments}

This study was supported by NASA under contract number NNC13TA84T.04. The computational resources were provided by the NASA Advanced Supercomputing (NAS) Division.

\section{References}

1. Lord Rayleigh, "The explanation of certain acoustical phenomena," Nature, Vol. 18 (455), 1878, pp.. 319-321.

2. Paxson, D.E. and Dougherty, K., "Ejector Enhanced Pulsejet Based Pressure Gain Combustors: An Old Idea With a New Twist," AIAA paper 2005-4216, July 2005.

3. Keller, J.O. and Bramlette, T.T., Dec, J.E. and Westbrook, C.J., "Pulse Combustion: The Importance of Characteristic Times," Combustion and Flame, Vol. 75, 1989, pp. 33-44.

4. Yungster, S., Paxson, D.E. and Perkins, H.D.," Computational Study of Pulsejet-Driven Pressure Gain Combustors at High-Pressure," AIAA paper 2013-3709, July 2013.

5. Yungster, S., Paxson, D.E. and Perkins, H.D.," Effect of Fuel Injection and Mixing Characteristics on PulseCombustor Performance at High-Pressure," AIAA paper 2014-3728, July 2014.

6. Yungster, S., Paxson, D.E. and Perkins, H.D.,"Parametric Study of Pulse Combustor-Driven Ejectors at High-Pressure," AIAA paper 2015-4190, July 2015.

7. Yungster, S., Paxson, D.E. and Perkins, H.D.,"Numerical Investigation of Shrouded Ejector-Enhanced Pulse Combustor Performance at High Pressure," Journal of Propulsion and Power, Vol. 33, No. 1, 2017, pp. 29-42.

8. Yungster, S., Paxson, D.E. and Perkins, H.D.,"Numerical Evaluation of an Ejector-Enhanced Resonant Pulse Combustor with a Poppet Inlet Valve and a Converging Exhaust Nozzle," AIAA paper 2016-4559, July 2016.

9. Offord, T., Miller, R. J., Dawson, J. R., Heffer, J. H., Mason, S. A., and Taylor, M., "Improving the Performance of a Valveless Pulse Combustor Using Unsteady Fuel Injection," AIAA Paper 2008-120, January 2008.

10. Yungster, S., "Numerical Study of Shock Wave/Boundary Layer Interactions in Premixed Combustible Gases," AIAA Journal, Vol. 30, No. 10, 1992, pp. 2379-2387.

11. Spalart, P. R. and Allmaras, S. R., A One-Equation Turbulence Model for Aerodynamic Flows, La Recherche Aerospatiale, Vol. 1, 1994, pp. 5-21.

12. Ajmani, K., Kundu, K. and Penko, P.F., "A Study on Detonation of Jet-A Using a Reduced Mechanism," AIAA paper 2010-1515, January 2010.

13. Ajmani, K., Kundu, K. and Yungster, S., "Assessment of Reduced Kinetics Mechanisms for Combustion of Jet Fuel in CFD Applications," AIAA 2014-3662, July 2014.

14. Ajmani, K., Mongia, H., and Lee, P., "Parametric Design of Injectors for LDI-3 Combustors", AIAA paper 2015-3785, July 2015. 
15. Ajmani, K., Mongia, H., and Lee, P., "CFD computations of emissions for LDI-2 combustors with simplex and airblast injectors", AIAA paper 2014-3529, July 2014.

16. Yungster, S. and Radhakrishnan, K., A Fully Implicit Time Accurate Method for Hypersonic Combustion: Application to Shock-Induced Combustion Instability. Shock Waves, Vol. 5, 1996, pp. 293-303.

17. Yungster, S. and Radhakrishnan, K., Pulsating one-dimensional detonations in hydrogen-air mixtures. Combustion Theory and Modeling, Vol. 8, 2004, pp. 745-770.

18. Van Heerbeek, P.A.,"Mathematical Modelling of a Pulse Combustor of the Helmholtz type," M.Sc. Thesis, Delft University of Technology, 2008.

19. Chu, B-T., "On th Energy Transfer to Small Disturbances in Fluid Flow (Part I)," Acta Mechanica Vol. 1, No. 3, 1965, pp. 215-234.

20. Blackburn, R.J. and Miller, R.J., "The Rayleigh Efficiency of Pressure Gain Combustors," Journal of Propulsion and Power, Vol. 33, No. 1, 2017, pp. 51-61.

21. Anand,V., Jodele, J., Knight, E., Prisell, E., Lyrsell, O. and Gutmark, E.,"Dependence of Pressure, Combustion and Frequency Characteristics on Valved Pulsejet Combustor Geometries," Flow Turbulence and Combustion, Vol. 100, No. 3, 2018, pp. 829-848.

22. Yungster, S., and Perkins, H. D., "Parametric Study of the Pulse Detonation Rocket- Based Combined-Cycle Engine," 58th Joint Army Navy NASA Airforce Propulsion Meeting [CD-ROM], The Johns Hopkins Univ., Chemical Propulsion Information Analysis Center (CPIAC), Arlington, VA, Apr. 2011.

23. Keller, J.O., Barr, P.K. and Gemmen, R.S., "Premixed Combustion in a Periodic Flow Field. Part I, Experimental Investigation," Combustion and Flame, Vol. 99, No. 1, 1994, pp. 29-42.

\section{Appendix}

\begin{tabular}{|c|c|c|c|c|c|c|c|}
\hline $\begin{array}{c}\text { Case } \\
\text { Number }\end{array}$ & $\begin{array}{c}\text { Bypass } \\
\text { ratio, } \boldsymbol{\beta}\end{array}$ & $\begin{array}{c}\text { Frequency } \\
\text { (Hz) }\end{array}$ & $\phi$ & $\begin{array}{c}\text { RPC } \\
\text { length } \\
\text { (in.) }\end{array}$ & $\begin{array}{c}\text { Ejector } \\
\text { length } \\
\text { (in.) }\end{array}$ & $\begin{array}{c}\text { Valve } \\
\text { opening time } \\
\text { (ms) }\end{array}$ & $\begin{array}{c}\text { Fuel injection } \\
\text { pressure (bar) }\end{array}$ \\
\hline 1 & 0.66 & 371.0 & 0.77 & 19.3 & 14.2 & 1.0 & 10.5 \\
\hline 2 & 1.61 & 359.0 & 0.76 & 19.3 & 14.2 & 1.0 & 10.5 \\
\hline 3 & 1.63 & 322.9 & 0.66 & 19.3 & 14.2 & 1.0 & 10.3 \\
\hline 4 & 1.80 & 358.5 & 0.75 & 19.3 & 12.2 & 1.0 & 10.5 \\
\hline 5 & 1.90 & 333.5 & 0.65 & 19.3 & 10.2 & 1.0 & 10.5 \\
\hline 6 & 1.88 & 346.6 & 0.60 & 17.3 & 12.2 & 1.0 & 10.5 \\
\hline 7 & 1.81 & 353.0 & 0.68 & 17.3 & 12.2 & 1.0 & 10.8 \\
\hline 8 & 1.97 & 392.2 & 0.80 & 17.3 & 12.2 & 1.0 & 11.2 \\
\hline 9 & 2.00 & 373.5 & 0.60 & 15.3 & 12.2 & 1.0 & 10.8 \\
\hline 10 & 1.85 & 377.5 & 0.62 & 15.3 & 12.2 & 1.0 & 11.0 \\
\hline 11 & 1.68 & 453.1 & 0.87 & 15.3 & 10.2 & 0.75 & 10.8 \\
\hline 12 & 1.60 & 403.3 & 0.75 & 15.3 & 10.2 & 0.75 & 10.4 \\
\hline 13 & 2.31 & 403.3 & 0.56 & 13.3 & 12.2 & 1.0 & 10.8 \\
\hline
\end{tabular}

